\title{
The amphiphysin family of proteins and their role in endocytosis at the synapse
}

\author{
Patrick Wigge and Harvey T. McMahon
}

\begin{abstract}
Clathrin-mediated endocytosis at the plasma membrane is a major pathway of synaptic vesicle recycling in neurones, but little is known about the molecular machinery that orchestrates the process. The amphiphysin protein has recently emerged into the limelight since its discovery in 1992 as a synaptic vesicle-associated protein. It was subsequently found to interact in vitro with the GTPase dynamin through its SH3 domain. However, only in the past year has its role in endocytosis been confirmed, with the demonstration that the introduction of dominant-negative-acting $\mathrm{SH} 3$ domains into living cells causes a potent blockade of clathrin-mediated endocytosis.This, together with the discovery by several groups of a second nerve terminal-enriched amphiphysin isoform, and the finding that the two proteins heterodimerize, further suggests that the amphiphysins are closely connected with dynamin-mediated vesicle budding. This review summarizes current views in the field, and draws on data that suggest intriguing alternative roles - including possible involvement in the cytoskeleton and in tumour suppression - for certain members of the amphiphysin family.
\end{abstract}

Trends Neurosci. (1998) 21, 339-344

\begin{abstract}
T IS IMPORTANT to understand how, in the brain, the millions of neurones communicate with each other across a multitude of interconnecting synapses. After the release of neurotransmitter by exocytosis, synaptic vesicles must be continuously re-formed from the presynaptic plasma membrane. A major component of this vesicle recycling occurs by a clathrin-mediated pathway in which the GTPase dynamin plays a crucial role ${ }^{1-4}$. This is probably a specialised, 'Ferrari' version of a general clathrin-mediated pathway occurring in all cells ${ }^{5}$. Regardless of cell type, endocytosis is thought to involve a series of steps beginning with the clustering of receptors at specific sites of the plasma membrane, regions that later turn into clathrin-coated pits. They do this by recruiting cytosolic AP-2 adaptor complexes through their cytoplasmic tails. In neurones, synaptotagmin is one likely candidate for such a receptor ${ }^{6}$. Clathrin triskelia are in turn moved to the membrane, and polymerize to form a curved polygonal lattice of hexagons and pentagons (analogous to a football) that provides the mechanical scaffold for the coated pit $^{7}$. Dynamin then self-assembles into ring-like structures collaring the neck of the invaginating, constricted coated vesicle, an event that leads ultimately to its pinching-off ${ }^{8,9}$. The first insights into how dynamin might be working at the synapse were derived from careful analyses of fruit-flies carrying a mutation in shibire, the Drosophila homolog of the gene for dynamin ${ }^{10}$. These flies are rapidly and reversibly paralysed at the restrictive temperature because of a block at a late stage of endocytosis. Nerve terminals show a dramatic depletion of synaptic vesicles and an accumulation of partially endocytosed, clathrin-coated pit profiles collared by electron-dense rings resembling those formed by dynamin in solution $^{9}$. Together with experiments in fibroblasts ${ }^{11,12}$, the
\end{abstract}

genetics and biochemistry strongly indicate that dynamin serves a mechanochemical function essential for vesicle budding. The current, favoured hypothesis for dynamin action (although not without its critics $^{13}$ ), holds that it acts as a 'pinchase', which, upon GTP hydrolysis, undergoes a conformational change that leads to closure of the rings and vesicle fission.

The specific targeting of dynamin to clathrin-coated pits, and the resultant triggering of its enzyme activity, must be regulated both spatially and temporally. Compelling evidence now suggests that the amphiphysins, a group of brain-enriched dynaminbinding proteins, play important role(s) in these processes. Although dynamin and endocytosis are subjects that have been reviewed extensively before ${ }^{3,5,14-16}$, this review will be dedicated especially to the amphiphysin family.

Role of the amphiphysin-dynamin interaction in endocytosis

Amphiphysin 1 (Amph1) was first identified in 1992 as a brain protein that was partially associated with synaptic vesicles ${ }^{17}$. Now the amphiphysin family is known to consist of several isoforms in mammals, yeast and nematodes (Fig. 1).

An interaction between the SH3 domain of Amph1 and the proline-rich domain of dynamin first suggested an involvement of Amph1 in endocytosis of synaptic vesicles $^{20}$. However, dynamin forms many proteinprotein interactions in vitro that do not necessarily occur in vivo ${ }^{15}$, so it was important to support this by experiments on its function. This was done by use of recombinant SH3 domains as 'dominant-negative' versions of Amph1 in living cells. Microinjection of such a purified SH3 domain into the giant reticulospinal synapse of the lamprey led to a massive block in endocytosis of
Patrick Wigge and Harvey T. McMahon are at the Neurobiology Division, MRC

Laboratory of Molecular Biology, Hills Road, Cambridge, UK $\mathrm{CB} 22 \mathrm{QH}$. 

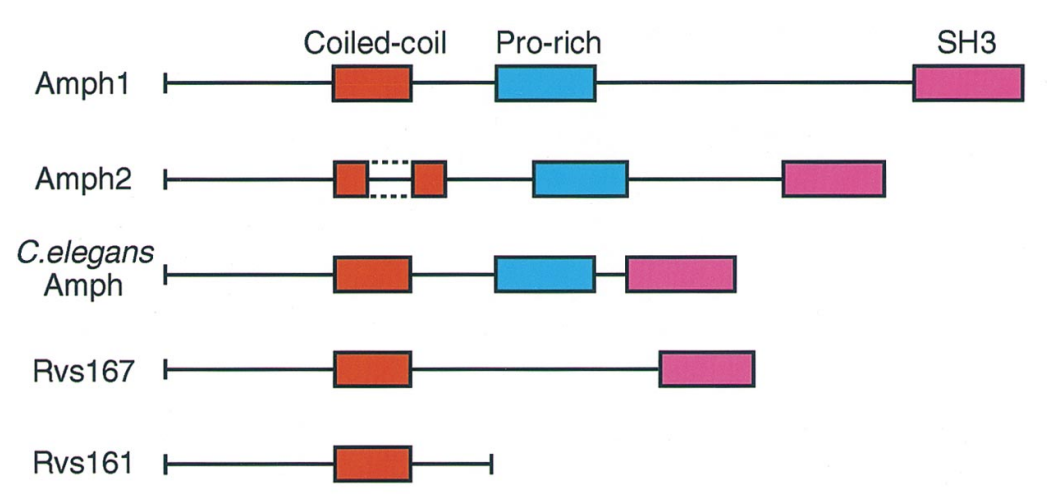

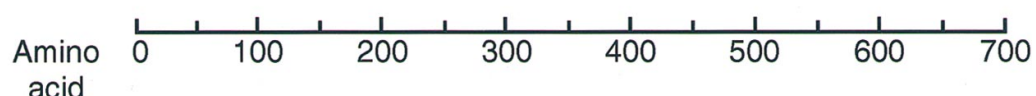

Fig. 1. Amphiphysin (Amph) family members in vertebrates, nematodes and yeast. Domain diagram of the amphiphysin family, including the yeast homologues Rvs161 and Rvs167, as defined by a similar underlying domain structure. For Amph1 and Amph2, the longest forms of these proteins are shown (corresponding to the brain forms); the many splice forms are not shown (but see other studies ${ }^{22,23}$ ). Although the yeast and mammalian proteins are only weakly related in amino acid sequence, they share a highly acidic, hydrophilic nature. All five proteins contain a predicted coiled-coil motif of six heptad repeats near the N-terminus, which, in the case of Amph2, is interrupted by an exon that is spliced out in most Amph2 isotypes (dotted line). It is probably through this coiled-coil domain that Amph1 and Amph2 heterodimerize with each other. All except Rvs 161 share a conserved, C-terminus SH3 domain. Linking the coiled-coil and SH3 domain is a poorly conserved, proline-rich linker region. The yeast homologues lack this proline-rich stretch, but Rvs167 has a corresponding region rich in glycine, proline and alanine (hence named the GPA region). In addition to the two isoforms identified in budding yeast, the sequence of an Rvs161 homologue in fission yeast, Rvs161-like protein, has recently been deposited in the database.

synaptic vesicles, and an accumulation at the plasma membrane of clathrin-coated pits, which were mostly devoid of dynamin collars ${ }^{21}$. In COS-7 fibroblasts, transfection of the SH3 domain of Amph1 led to a potent block of receptor-mediated endocytosis, as shown by transferrin uptake ${ }^{22}$. Importantly, the SH3 domains of Grb2, spectrin or PLC $\gamma$, all of which interact with dynamin in vitro, failed to exert any effect in vivo ${ }^{22}$. Coexpression of dynamin rescued the SH3domain block, suggesting that dynamin is the cellular target. The common interpretation of these experiments was that the SH3 domain of Amph1 acted by disrupting dynamin-amphiphysin complexes at clathrin-coated pits. This suggests that Amph1 might have an important role in the recruitment of dynamin to sites of endocytosis. Although far from representing a model neurone, COS-7 cells do indeed express Amph1 at a low level (showing that it is not a brainspecific protein) and, furthermore, they have been used in the past to help infer the function of dynamin in vivo as well ${ }^{11,23}$.

The SH3 domain of Amph1 is also a major target of the autoantibodies in some cases of Stiff-Man syndrome ${ }^{24,25}$. This is a rare autoimmune disorder characterised by several neurological symptoms, including muscle spasms. Interestingly, these antibodies are targeted predominantly to the SH3 domain ${ }^{26}$.

Dynamin is not the only in vitro binding partner for Amph1. The presynaptic $145 \mathrm{kDa}$ protein, synaptojanin, is an enzyme with a $5^{\prime}$ phosphatase activity that acts on a number of different phospholipids, including phosphatidylinositol-4,5-bisphosphate ${ }^{27}$. Synaptojanin contains a polyproline domain that is highly similar to that of dynamin at its C-terminus, and has been found to interact with amphiphysin, again via its $\mathrm{SH} 3$ domain $^{27}$. It may thus act to regulate negatively dynamin recruitment to clathrin-coated pits in either of two ways. First, its occupation of SH3 domains at the membrane would be predicted to prevent dynamin's recruitment via amphiphysin. Second, dynamin has a PH domain, which has high affinity for $\mathrm{PI}(4,5) \mathrm{P}_{2}$ but not for its hydrolysis product, PI(4)P. Dynamin's interaction with phospholipids at the membrane, as well as with amphiphysin, could therefore be modulated by the actions of this important lipid phosphatase.

Amphiphysin 1 is found predominantly in the brain, as are dynamin I and many other proteins associated with cycling of synaptic vesicles. By analogy with dynamin, one would expect to find less-predominant isoforms (such as dynamin II) functioning in other, constitutive endocytotic events. How accurate is this hypothesis in the context of the amphiphysins? The second amphiphysin gene in mammals, amphiphysin 2 (of which various splice variants have been alternatively named BIN-1, SH3P9, BRAMP-2 or ALP-1) has now been cloned by eight independent groups ${ }^{18,19,28-32}$. The largest isoform, a $95 \mathrm{kDa}$ protein, is by far the major type present in the brain, and appears to be brain-specific. However, the fact that there are smaller isotypes expressed ubiquitously ${ }^{19,30}$, some in cell type-specific patterns ${ }^{18}$, suggests that the amphiphysin family is indeed capable of acting in more general clathrin-mediated endocytosis outside of the brain.

In the brain, Amph2 shares a very similar (if not identical) subcellular distribution to that of Amph1, being highly concentrated in nerve terminals as confirmed by electron microscopy ${ }^{19,31}$. Significantly, Amph1 and Amph 2 can be co-immunoprecipitated as a complex. In the absence of any other proteins, the two amphiphysins can form a stable heterodimer, and this is in fact the predominant form in which they are found in the brain. Because Amph2 has an SH3 domain that is highly similar to that of Amph1, and can also interact with dynamin I, it has been suggested that the heterodimer represents a form of amphiphysin that would drive the efficient recruitment of multiple dynamin molecules to sites of fast endocytosis at nerve terminals ${ }^{19}$. A high local concentration of dynamin at clathrin-coated pits would favour the formation of rings around the vesicle neck, in preparation for the budding step.

In order for endocytosis to continue, however, it is important that dynamin does not remain permanently bound to amphiphysin. Thus, it should be possible to 'switch off' the dynamin-amphiphysin interaction as soon as dynamin has been recruited to the clathrin-coated pit. Recent experiments from our laboratory suggest that the dissociation of amphiphysin from dynamin is coupled closely to the state of oligomerization of dynamin. The SH3 domains of both Amph1 and Amph2, unlike all other SH3 domains tested, favour the disassembly of dynamin rings in vitro probably by sequestering the dissociated form $^{33}$. One might argue that this is more compatible with a post-budding role of amphiphysin in dynamin disassembly (that is, the recycling of dynamin after the fission step) than a role in dynamin recruitment prior to the budding step. However, microinjection of a dynamin peptide (which blocks the interaction of 
dynamin with both Amph1 and Amph2) into presynaptic terminals of lamprey resulted in a marked absence, not an accumulation, of dynamin rings at clathrin-coated pits $^{21}$. If then, amphiphysin functions in the recruitment step, and is not a recycling factor, how does its peculiar effect on multimerization of dynamin fit into the picture? Amphiphysin might specifically recruit dynamin in its dissociated state (dimers $^{34}$ or tetramers ${ }^{35}$ ), and maintain it in this form just at those sites of the membrane where it is needed. Viewed in this way, sequesteration of dynamin at a high local concentration would be a prerequisite for self-assembly into rings at the clathrin-coated pit. As soon as dynamin has multimerized into rings this would trigger the simultaneous dissociation of amphiphysin heterodimers, since ring formation and amphiphysin binding are mutually exclusive ${ }^{33}$. This might explain the recent observations of Bauerfeind et al., who find amphiphysin to be associated with coated pit intermediates but apparently not as integral components of the dynamin ring ${ }^{36}$. And it may go some way to explain why other SH3 domains do not affect endocytosis when overexpressed in fibroblasts ${ }^{22}$ : while Grb2, spectrin and PLC $\gamma$ all can interact with dynamin in vitro, they cannot mimic the SH3 domains of Amph1 or Amph2 in preventing dynamin self-assembly.

\section{Potential interactions with other elements of the endocytotic apparatus}

As well as a C-terminal SH3 domain, both Amph1 and Amph2 contain sites for interaction with other targets that implicate them further in clathrin-mediated endocytosis. An important factor in the formation of clathrin-coated pits is the plasma-membrane adaptor complex AP-2, a heterotetrameric complex that has recently been shown to interact with both Amph1 and Amph2 via its $\alpha$ subunit ${ }^{19,30,37}$. This is mediated through a region that is distinct from the SH3 domain of amphiphysin ${ }^{20}$, and indeed, AP-2 and dynamin can interact with Amph1 simultaneously ${ }^{22}$. Although the interaction of the amphiphysins with AP-2-adaptor complexes remains to be demonstrated in vivo, it is tempting to speculate that it could represent an important means by which normally soluble amphiphysin heterodimers are localised to the presynaptic plasma membrane.

Affinity purification of brain extracts using recombinant amphiphysins as 'bait' has led to the identification of a further potential binding partner, clathrin $^{31,38}$. This appears to be a direct, specific interaction that is mediated by two sequences conserved in the central regions of both Amph1 and Amph2 (Ref. 39). Although mediated by different regions of the amphiphysin molecule, the interactions of clathrin and of dynamin appear to be mutually exclusive, an observation that might help to explain some of the specificity of dynamin recruitment to the necks of forming clathrin-coated vesicles ${ }^{38}$. Clathrin-binding to Amph2 is also selective and might be a special feature of the role of amphiphysin in the nervous system, since several non-neuronal splice forms do not bind to clathrin at all ${ }^{39}$.

Figure 2 summarizes the protein-protein interactions discussed so far that are thought to play a role in budding of clathrin-coated vesicles. According to this scheme, the amphiphysins lie at the very heart of this web of protein-protein interactions and, through associations

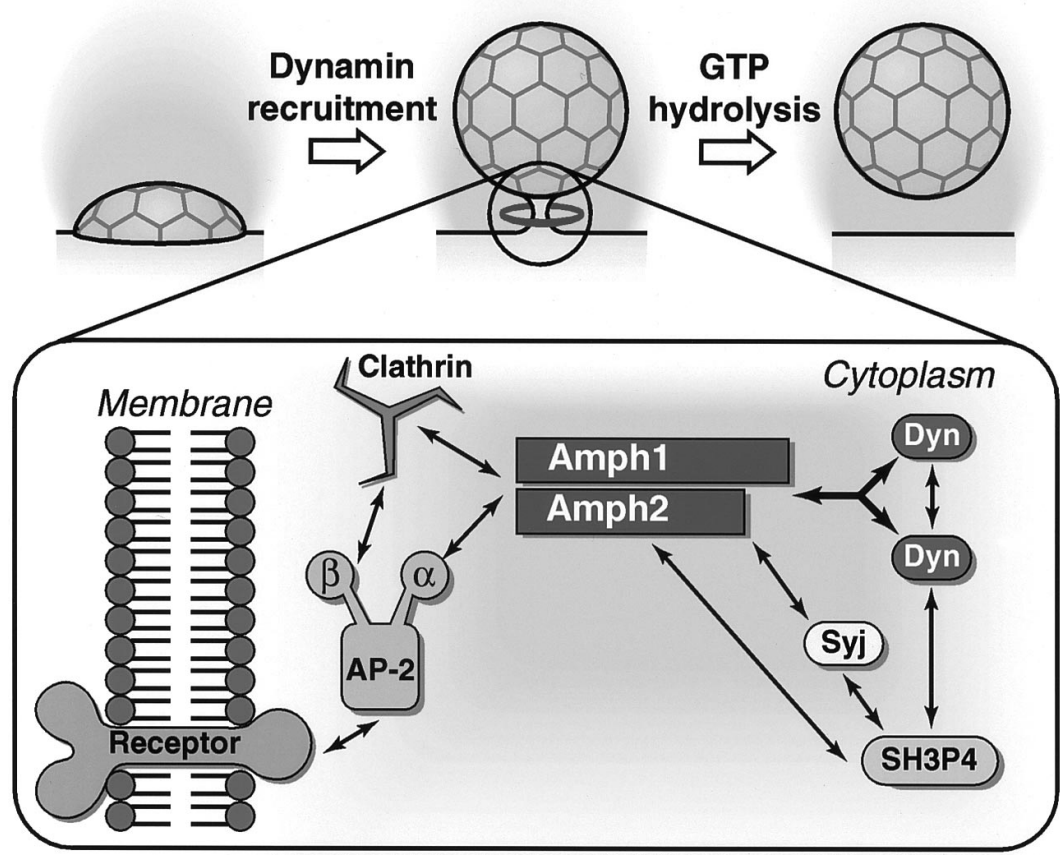

Fig. 2. How amphiphysins might act in endocytosis at the nerve terminal. Hypothetical scheme showing the web of protein-protein interactions that amphiphysin heterodimers are capable of forming at the nerve terminal, and how they might participate in the recruitment of dynamin to sites of endocytosis of synaptic vesicles. It is important to note that not all of these interactions have been confirmed in vivo. However, experiments with dominant-negative recombinant versions of amphiphysin described in this review provide in vivo evidence for the importance of the amphiphysin-dynamin interaction (bold arrows). Of all the amphiphysin ligands depicted, it is interesting that none seems to interact in an isoform-specific manner; dynamin, AP-2, clathrin and synaptojanin have all been found to bind to both Amph1 and Amph2. The theoretical question of what extra function is provided by the heterodimer (as compared with, for example, homodimers) is therefore still somewhat of a puzzle.

with each other and with dynamin, synaptojanin, clathrin, and AP-2 complexes are well positioned to perform an essential function in endocytosis at the nerve terminal. Of course, not all of these interactions would necessarily take place at the same time; for example, synaptojanin and dynamin compete with one another for binding to the SH3 domain of amphiphysin. Finely tuned regulatory events, such as dephosphorylation of several endocytosis proteins (Box 1), are likely to affect the order in which these interactions occur, and ultimately control the process.

\section{Clues from the molecular ancestors of the amphiphysins: the yeast Rvs family}

Endocytosis can also occur in yeast through a receptor-mediated pathway. Although many mammalian proteins have yeast homologues that serve similar functions, the extent to which mechanisms of endocytosis have been conserved through evolution is less clear. It is important to know, therefore, whether the amphiphysins have the same function in S. cerevisiae as they do in other organisms?

There are two genes in budding yeast, rvs161 and rvs167, that share homology with the vertebrate amphiphysins (see Fig. 1). They were named according to the phenotype of yeast strains containing mutations in either of these genes, namely a reduced viability upon nutrient starvation. This is thought to be due to defects in regulation of the cell cycle in these yeast strains - when dividing exponentially, they are unable to enter into stationary phase ${ }^{40}$. What connections, if any, do the Rvs family have with endocytosis? 


\section{Box I. Phosphorylation-dephosphorylation cycling of endocytosis proteins: a potential regulatory mechanism?}

Synaptic vesicle exocytosis has a tight calcium-dependence, occuring in milliseconds after the opening of calcium channels at the active zone. Synaptotagmin has been implicated as the calcium sensor for exocytosis ${ }^{\mathrm{a}}$, but for a long time it has been somewhat of a mystery whether a similar calcium 'sensor' exists for endocytosis. Recently, experiments using a lipophilic fluorescent dye as a means to follow endocytosis in isolated nerve terminals ${ }^{\mathrm{b}}$, together with membrane-capacitance studies in adrenal chromaffin cells ${ }^{\mathrm{c}}$, have provided evidence for the importance of calcium in synaptic vesicle retrieval.

A candidate receptor for calcium in both of the above studies was shown to be calcineurin, by use of the inhibitor cyclosporin A. Calcineurin is a calcium- and calmodulin-dependent phosphatase which had previously been suggested to regulate vesicle cycling through its action on dynamin ${ }^{\mathrm{d}}$, and indeed is enriched in nerve terminals. The calcineurin-sensitive pathway in nerve terminals is dependent on the interaction of dynamin with amphiphysin $^{\mathrm{b}}$, and thus might be clathrin-mediated. Of the two endocytosis pathways identified in chromaffin cells (a compensatory pathway occurring in seconds, and an ultra-fast 'excess retrieval' pathway) only the former is affected by calcineurinc, and again this pathway might represent clathrin-mediated retrieval, since it has a slower time-constant and is selectively abolished on whole-cell dialysis where the cytosolic components would be lost.

What is the target of calcineurin? Dynamin ${ }^{\mathrm{d}}$, amphiphysins- 1 and -2 (Refs b,e,g) as well as synaptojanin ${ }^{\mathrm{f}}$ are phosphorylated in resting nerve terminals, and are rapidly dephosphorylated, in parallel, upon stimulation of exo/endocytosis. Cyclosporin A prevents the dephosphorylation of all these proteins $\mathrm{s}^{\mathrm{b}, \mathrm{g}}$, thus showing that the phosphorylation states of all four endocytosis proteins are regulated by a common phosphatase, calcineurin. On repolarization of nerve terminals, dynamin, the amphiphysins, and synaptojanin are all rephosphorylated, a process dependent on protein kinase C (Refs e,h).

But how does protein dephosphorylation act in the initiation, or regulation, of endocytosis? Only for dynamin I has phosphorylation been demonstrated to change the protein's activity. In solution, its intrinsic GTPase activity is high when phosphorylated ${ }^{\mathrm{h}}$, but inhibited about 10 -fold when dephosphorylated by calcineurin ${ }^{\mathrm{d}}$.
How would this be expected to affect the process? During stimulation of synaptic vesicle endocytosis, dephosphorylation of dynamin I would inhibit its GTPase, increasing the pool of dynamin-GTP at the expense of the GDP-bound form of the protein. This rapidly triggered dephosphorylation might therefore allow a close coupling of dynamin ring formation to cycles of exocytosis and endocytosis. However, phosphorylation is by no means the only factor influencing the GTPase activity of dynamin, since acidic phospholipids ${ }^{\mathrm{i}}$ microtubules and various SH3 domains ${ }^{\mathrm{k}}$ have all been reported to activate this enzyme activity in low salt. All of these activities possibly occur by the enhancement of dynamin multimerization ${ }^{1}$ and so these observations should be interpreted with caution. Unlike dynamin, the amphiphysins do not possess an enzyme activity that could be regulated by phosphorylation. How, then, are they affected by it? The answer to this has turned out to be elusive, as neither their interaction with dynamin nor with AP-2 adaptors appear to be regulated by phosphorylation. An interesting hint that might give some more insight into the function of Amph2 is the observation that compared to Amph1, dynamin and synaptojanin, Amph2 is dephosphorylated more extensively during depolarization ${ }^{\mathrm{b}, \mathrm{e}}$.

\section{References}

a Brose, N. et al. (1992) Science 256, 1021-1025

b Marks, B. and McMahon, H.T. (1998) Curr. Biol. 8, 740-749

c Engisch, K.L. and Nowycky, M.C. (1998) J. Physiol. 506, $591-608$

d Liu, J-P., Sim, A.T. and Robinson, P.J. (1994) Science 265, 970-973

e Wigge, P. et al. (1997) Mol. Biol. Cell 8, 2003-2015

f McPherson, P.S. et al. (1994) J. Biol. Chem. 269, 30132-30139

g Bauerfeind, R., Takei, K. and DeCamilli, P. (1997) J. Biol. Chem. 272, 30984-30992

h Robinson, P.J. et al. (1993) Nature 365, 107-108

i Tuma, P.L., Stachniak, M.C. and Collins, C.A. (1993) J. Biol. Chem. 268, 17240-17246

i Herskovits, J.S. et al. (1993) Proc. Natl. Acad. Sci. U. S. A. 90 $11468-11472$

k Gout, I. et al. (1993) Cell 75, 25-36

1 Warnock, D.E., HInshaw, J.E. and Schmid, S.L. (1996)

J. Biol. Chem. 271, 22310-22314
Although Rvs167p contains an SH3 domain, it is unlikely to play the same role as Amph1 or Amph2. No dynamin homologue that is capable of acting at the plasma membrane has been identified in yeast, indicating that the 'pinchase' step is governed by an entirely different protein, perhaps $\operatorname{myosin}^{41}$. However, other features of the amphiphysins seem to be shared by the yeast proteins. Genetic and biochemical evidence strongly suggest that Rvs161p and Rvs167p form a heterodimer ${ }^{42,43}$. Singly mutant strains defective in either rvs161 or rvs167 exhibit the full mutant phenotype characteristic of doubly mutant rvs161-:rvs167- yeast strains. Furthermore, both loci are suppressed by the same set of SUR genes ${ }^{44}$.

Even though the Rvs proteins cannot interact with a plasma-membrane homologue of dynamin, the phenotype of the mutant strains is indicative of a role in endocytosis. Both receptor-mediated and fluidphase uptake are blocked ${ }^{45}$. Other phenotypic symptoms suggest that at least part of the endocytotic defect can be attributed to a noticeable disruption of the peripheral cytoskeleton. As well as showing a mislocalization of cortical actin patches on the plasma membrane ${ }^{45}$, rvs mutant yeast cells exhibit abnormal polarity and an uneven cell size ${ }^{46}$. Furthermore, the SH3 domain of Rvs167p was identified in a two-hybrid screen for actin-binding proteins ${ }^{47}$, suggesting a molecular basis for these cytoskeletal effects. While it seems likely that in yeast the Rvs proteins have interrelated effects on the actin cytoskeleton and on endocytosis, no direct biochemical interaction between Amph1/Amph2 and actin has yet been demonstrated.

\section{Other suggested roles - distinct from, or connected to, endocytosis?}

Although direct evidence for the involvement of amphiphysin in the mammalian actin cytoskeleton is lacking, a recent study has reported that Amph2 protein localises to areas of actin fibres in brain and in skeletal muscle ${ }^{33}$. In the brain it was reported to be 
concentrated in the cortical cytomatrix of axon initial segments and nodes of Ranvier and to have a distinct distribution from that of Amph1. However, this finding is at odds with two other studies, which established that the brain form of Amph2, as for Amph1, is enriched in nerve terminals ${ }^{19,31}$ and forms heterodimers with Amph1 (Ref. 19). Outside the brain, it is possible that other splice forms of Amph2 have alternative subcellular distributions. Indeed, in skeletal muscle a smaller $(50 \mathrm{kDa})$ Amph2 protein is expressed at high levels, and probably forms homodimers, since no Amph1 isotype is expressed at corresponding levels. Here, the protein was found by light microscopy to be present at $\mathrm{T}$ tubules, where it co-localizes with the cytomatrix-specific ankyrin3 (Ref. 32). Its presence at these sites could reflect either an involvement in endocytosis from the plasma membrane, since clathrin is present at $\mathrm{T}$ tubules $^{48}$, or, alternatively, a generalized role in the physiology of the peripheral cytoskeleton $^{32}$.

Perhaps the most surprising role put forward for Amph2 is an involvement in tumour suppression. In a two-hybrid screen for Myc-interacting proteins, a splice variant of Amph2 was isolated, and named BIN-1 (Ref. 28). This is almost identical to the brain form of Amph2, apart from an insertion of an additional short exon, which in fact encodes a nuclear-localization signal. BIN-1 and Myc were shown to interact in vitro, but could not be co-immunoprecipitated. A functional significance for the interaction with Myc was suggested by results of a co-operativity assay indicating that BIN-1 can inhibit focus formation by the tumour promoters MYC and RAS, a property dependent on the Mycbinding domain ${ }^{28}$. Interestingly, half of all tumour cell lines (including breast cancers) had greatly reduced levels of BIN-1 transcript. A link has been suggested with the cell cycle defects in rvs mutant yeast, but neither Rvs161 nor Rvs167 possesses a Myc-binding domain or a nuclear localization signal, so it appears unlikely that the same Myc-dependent mechanisms could explain this phenotype.

A very recent study reported an interaction between the tyrosine kinase c-Abl and ALP-1, an Amph2 splice variant with a broad tissue distribution ${ }^{49}$. The interaction occurs through the SH3 domain of ALP-1. Coexpression of ALP-1 and c-Abl led to an alteration of cell morphology; from this, it has been suggested that ALP-1 might help to mediate the cytoskeletal role of Abl. While this is very intriguing, many SH3-domain proteins are known to bind to Abl; coexpression of some of these other potential effectors with Abl would help to show how specific and physiologically important the interaction with Abl really is.

These studies add complexity to the Amph2 story. Are these diverse functions related? The amphiphysins certainly appear to interact with several potential proteins, not all of which are implicated in endocytosis. The additional apparent involvement of the actin cytoskeleton in the function of amphiphysin is interesting but the data all suggest that this link might occur in different ways, through actin itself, through c-Abl, or possibly through components of the cytomatrix. One possible (though unlikely) explanation is that the amphiphysins are at the crossroads of multiple signalling pathways affecting (directly or indirectly) the cytoskeleton and regulation of the cell cycle as well as endocytosis. If this idea is consolidated, Amph2 could be added to the growing list of 'adaptor' proteins suspected of linkng endocytosis to signal-transduction events emanating from plasma-membrane receptors. Interestingly, one of the eight groups that have independently cloned Amph2 has shown that stimulation of PC12 cells with nerve growth factor (which activates signal-transduction events from its receptor, as well as its internalization) led to an increase in the amount of dynamin co-immunoprecipitated with Amph2 from these cells ${ }^{30}$.

Despite the alternative suggested roles for Amph2, the bulk of the available evidence supports a key role for the amphiphysins in endocytosis. To recap and briefly summarize: (1) dynamin is a stoichiometric binding partner for Amph1 and Amph2 in brain extracts; (2) SH3 domains of Amph sequester dynamin specifically in its dissociated form; (3) overexpression of Amph SH3 domains potently inhibits clathrinmediated endocytosis either in nerve terminals or in fibroblasts; (4) Amph is concentrated in nerve terminals where it is associated with clathrin-coated pit intermediates; (5) Amph1 and Amph2 are rapidly dephosphorylated when nerve terminals are stimulated, along with dynamin and synaptojanin; (6) immunocytochemistry shows that Amph2, like Amph1 and dynamin, are expressed at higher levels in a subset of nerve terminals ${ }^{19}$. These terminals are densely filled with synaptic vesicles, indicative of a high synaptic activity.

\section{Concluding remarks}

The data emerging from a combination of biochemical experiments and functional data provide persuasive evidence for the role of amphiphysins 1 and 2 in the recruitment of dynamin during endocytosis. It is puzzling why Amph2 should have been additionally implicated in so many other cellular processes. Future studies could solve the question of whether these putative functions are related, and of whether the amphiphysins are really multifunctional proteins. However, the dynamin recruitment model provides a testable hypothesis, a starting point for experiments to elucidate further the precise molecular machinery of synaptic-vesicle recycling. What role does phosphorylation play in regulating the amphiphysins' activity? How are the amphiphysins specifically targeted to the clathrin-coated pit? Furthermore, and perhaps of more pertinent interest to electrophysiologists, does amphiphysin recruit dynamin during the more rapid, 'kiss-and-run' pathway of endocytosis as well? The groundwork has only just been laid.

\section{Selected references}

1 Heuser, J.E. and Reese, T.S. (1973) J. Cell Biol. 57, 315-344

2 Maycox, P.R. et al. (1992) J. Cell Biol. 118, 1379-1388

3 DeCamilli, P. and Takei, K. (1996) Neuron 16, 481-486

4 Gonzalez-Gaitan, M. and Jackie, H. (1997) Cell 88, 767-776

5 Morris, S.A. and Schmid, S.L. (1995) Curr. Biol. 5, 113-115

6 Zhang, J.Z. et al. (1994) Cell 78, 751-760

7 Pearse, B.M.F. and Robinson, M.S. (1990) Annu. Rev. Cell Biol. 6, 151-171

8 Takei, K. et al. (1995) Nature 374, 186-190

9 Hinshaw, J.E. and Schmid, S.L. (1995) Nature 374, 190-192

10 Kosaka, T. and Ikeda, K. (1983) J. Neurobiol. 14, 207-225

11 Herskovits, J.S. et al. (1993) J. Cell Biol. 122, 565-578

12 Damke, H. et al. (1994) J. Cell Biol. 127, 915-934

13 Roos, J. and Kelly, R.B. (1997) Trends Cell Biol. 7, 257-259

14 Robinson, M.S. (1994) Curr. Opin. Cell Biol. 6, 538-544

15 Urrutia, R. et al. (1997) Proc. Natl. Acad. Sci. U. S. A. 94, 377-384

16 McClure, S.J. and Robinson, P.J. (1996) Mol. Memb. Biol. 13, 189-215 
17 Lichte, B. et al. (1992) EMBO J. 11, 2521-2530

18 Tsutsui, K. et al. (1997) Biochim. Biophys. Res. Comm. 236, $178-183$

19 Wigge, P. et al. (1997) Mol. Biol. Cell 8, 2003-2015

20 David, C. et al. (1996) Proc. Natl. Acad. Sci. U. S. A. 93, 331-335

21 Shupliakov, O. et al. (1997) Science 276, 259-263

22 Wigge, P., Vallis, Y. and McMahon, H.T. (1997) Curr. Biol. 7, 554-560

23 Okamoto, P.M., Herskovits, J.S. and Vallee, R.B. (1997) J. Biol. Chem. 272, 11629-11635

24 Folli, F. et al. (1993) New Engl. I. Med. 328, 546-551

25 DeCamilli, P. et al. (1993) J. Exp. Med. 178, 2219-2223

26 David, C., Solimena, M. and DeCamilli, P. (1994) FEBS Lett. 351, 73-79

27 McPherson, P.S. et al. (1996) Nature 379, 353-357

28 Sakamuro, D. et al. (1996) Nature Genet. 14, 69-77

29 Sparks, A.B. et al. (1996) Nature Biotech. 14, 741-744

30 Leprince, C. et al. (1997) J. Biol. Chem. 272, 15101-15105

31 Ramjaun, A.R. et al. (1997) J. Biol. Chem. 272, 16700-16706

32 Butler, M.H. et al. (1997) J. Cell Biol. 137, 1355-1367

33 Owen, D.J. et al. EMBO J. (in press)

34 Tuma, P.L. and Collins, C.A. (1995) J. Biol. Chem. 270, 26707-26714
35 Muhlberg, A.B., Warnock, D.E. and Schmid, S.L. (1998) EMBO J. 16, 6676-6683

36 Bauerfeind, R., Takei, K. and DeCamilli, P. (1997) J. Biol. Chem. 272, 30984-30992

37 Wang, L-H., Südhof, T.C. and Anderson, R.G.W. (1995) J. Biol. Chem. 270, 10079-10083

38 McMahon, H.T., Wigge, P. and Smith, C. (1997) FEBS Lett. 413, 319-322

39 Ramjaun, A.R. and McPherson, P.S. (1998) J. Neurochem. 70, 2369-2376

40 Crouzet, M. et al. (1991) Yeast 7, 727-743

41 Geli, M.I. and Riezman, H. (1996) Science 272, 533-535

42 Bauer, F. et al. (1993) Mol. Cell Biol. 13, 5070-5084

43 Sivadon, P., Crouzet, M. and Aigle, M. (1997) FEBS Lett. 417 , 21-27

44 Desfarges, L. et al. (1993) Yeast 9, 267-277

45 Munn, A. L. et al. (1995) Mol. Biol. Cell 6, 1721-1742

46 Sivadon, P. et al. (1995) Mol. Gen. Genet. 246, 485-495

47 Amberg, D.C., Basart, E. and Botstein, D. (1995) Struct. Biol. 2 , 28-35

48 Kaufman, S.J., Bielser, D. and Foster, R.F. (1990) Exp. Cell Res 191, 227-238

49 Kadlec, L. and Pendergast, A.M. (1997) Proc. Natl. Acad. Sci. U. S. A. 94, 12390-12395

\title{
Neuronal chemistry and functional organization in the primate visual system
}

\author{
Stewart H.C. Hendry and David J. Calkins
}

Beginning with the first step of visual processing and proceeding outward from that point, the neurons involved in different aspects of vision are distinct. Stated simply, neurons doing different things look different. They often display distinct morphological features and they usually express different molecules. In addition, neurons that perform a common function usually aggregate together to form recognizable layers or compartments that can be studied in isolation because they are neurochemically distinct. Here is found, then, a junction of two major domains in neuroscience research, as discovery of molecular diversity among neurons is exploited to study organization and function of the primate visual system.

Trends Neurosci. (1998) 21, 344-349

A sats A LEGACY OF SPERRY'S chemoaffinity hypothesis $^{1}$, the expectation 15 years ago was that the selective expression of molecules would lead to the discovery of markers specific for neuronal types ${ }^{2}$. For the nervous systems of invertebrates the discovery and use of type-specific markers have met with frequent success. Not so successful has been the application of molecular biology to an understanding of organization and function in the mammalian CNS. The problem is simple, as marker proteins or peptides specific for individual types of neurons have rarely been encountered. Yet when successful, most notably in studies of the primate visual system, the discovery of molecules unique to specific neuronal groups and the ability to see and study those groups in isolation have proven extremely powerful for addressing questions that are significant to organization and physiology.

These questions range from the role of identified neuronal types in the ability most primates possess to discriminate objects of different colors, to the basic division of labor in well-characterized regions of the visual system. Most successes have occurred for regions early in the visual pathway - the retina, lateral geniculate nucleus
(LGN) and the primary visual area (V1) - but recent findings suggest that much will be learned of higher-order areas of the visual cortex by the application of molecular biology to visual system function and organization.

\section{Type-specific markers in primate retina}

\section{Sense and nonsense}

Expectations for type-specific markers have been well met for the mammalian retina, where proteins or peptides are expressed by restricted populations of neurons (Table 1). These type-specific molecules divide themselves sharply into two groups: those that make sense and those that do not. The sensible group is the one in which the basic function of the cell type is a direct function of the molecule. In this context, the photoreceptors stand out by their expression of photopigments or opsins, because each type of cone and the rods absorb a restricted range of wavelengths as a direct result of the opsin each expresses ${ }^{3,17}$. Thus the precise function of a photoreceptor type is predictable on the basis of its expression of an opsin, and mutations in opsin genes produce predictable outcomes in spectral sensitivity and color discrimination ${ }^{4,18}$. 